Información farmacológica

RETIROS DEL MERCADO

REACCIONES ADVERSAS

USO RACIONAL

ENMIENDAS A LA ROTULACIÓN/ CAMBIOS DE FORMULACIÓN

\section{EVALUACIÓN DE NUEVOS PRODUCTOS}

POLÍTICAS DE MEDICAMENTOS
Se suspende la venta de sibutramina; se intensifica la fiscalización de la comercialización de la hierba de San Juan (Hypericum perforatum).

Reacciones hepatobiliares a los fármacos antituberculosos; efectos adversos cardiovasculares del rofecoxib; trastornos hidroelectrolíticos con las soluciones orales de fosfato sódico; epoyetina $\alpha$ y aplasia pura de la serie roja; trastornos hematológicos con la linezolida; acidosis láctica mortal en pacientes con sida tratados con antirretrovíricos; complicaciones tromboembólicas con el raloxifeno; tramadol y síndrome serotonínico; toxicidad pulmonar de la amiodarona; reacciones adversas neuropsiquiátricas al zolpidem; cistitis con el ácido tiaprofénico; reacciones adversas al levofloxacino.

Nuevos consejos para la prescripción de propionato de fluticasona; seguridad gastrointestinal de los antiinflamatorios no esteroideos.

Advertencias de uso en los prospectos del etanercept; advertencia sobre el uso de la oprelveKina en pacientes pediátricos; cambios en la información sobre la seguridad de Intron ${ }^{\circledR} \mathrm{A}$ (interferón $\alpha_{2 b}$ ); cambios en los prospectos de la pioglitazona y la rosiglitazona; solicitud de revisión del prospecto de Exelon $^{\circledR}$ (rivastigmina).

Usos y efectos indeseables de los nuevos hipoglucemiantes orales; la FDA aprueba el primer anticonceptivo hormonal en parches cutáneos.

III Conferencia Panamericana sobre Armonización de la Reglamentación Farmacéutica; la OMS lanza la primera estrategia mundial sobre medicina tradicional y alternativa; la contracepción de emergencia estará disponible sin necesidad de prescripción médica.

\section{RETIROS DEL MERCADO}

Se suspende la venta de sibutramina (Italia)

El 6 de marzo de 2002 las autoridades italianas suspendieron temporalmente la autorización de comercialización en ese país de todos los medicamentos que contengan sibutramina y han sometido el asunto al Secretariado de la Agencia Europea de Evaluación del Medicamento (European Medicines Evaluation Agency: EMEA), para que realice una evaluación exhaustiva de los riesgos y beneficios del fármaco. La venta de sibutramina se aprobó en Italia en abril de
2001 y desde entonces se han notificado a las autoridades de ese país 50 reacciones adversas, 7 de la cuales se consideraron graves y 2 fueron mortales. Las más frecuentes fueron la taquicardia y la hipertensión. Las dos muertes se debieron a arritmia y paro cardíaco. Varios países europeos (Alemania, Dinamarca, España, Finlandia, Francia, Holanda, Portugal, Reino Unido y Suecia) han informado al público de la suspensión de la comercialización de la sibutramina en Italia. Alemania, Francia y el Reino Unido han anunciado que están examinando el problema, pero no han retirado el fármaco del mercado.
Se intensifica la fiscalización de la comercialización de la hierba de San Juan (Hypericum perforatum) (Brasil)

La hierba de San Juan (Hypericum perforatum) es un agente fitoterapéutico de uso popular en el tratamiento de las inflamaciones cónicas del estómago, hígado, vesícula biliar y riñones, afecciones ginecológicas, ansiedad, tensión nerviosa, trastornos de la menopausia y síndrome premenstrual, a pesar de que ninguna de estas supuestas indicaciones ha sido comprobada científicamente hasta la fecha. Por este motivo, la única indicación aprobada por la Agencia Nacional de 
Vigilancia Sanitaria (ANVISA) de Brasil, respaldada por pruebas clínicas, es el tratamiento de la depresión leve a moderada. Informes recientes de agencias de otros países señalan la existencia de importantes interacciones entre los productos que contienen hierba de San Juan y diferentes fármacos que requieren prescripción médica, como la ciclosporina, digoxina, anticonceptivos orales, teofilina, warfarina e indinavir. Dichas interacciones pueden reducir los efectos de estos fármacos o hacer que produzcan efectos tóxicos, en especial en el caso de la ciclosporina, digoxina, anticonceptivos orales, teofilina y warfarina. Esto ha llevado a la ANVISA a promulgar una resolución (RE No. 357, de 28 de febrero de 2002) de aprehensión de los productos que contengan hierba de San Juan y no dispongan de una etiqueta roja que diga "De venta con prescripción médica", así como de aquellos que no estén registrados en la ANVISA.

\section{REACCIONES ADVERSAS}

\section{Reacciones hepatobiliares a los fármacos antituberculosos (Canadá)}

La asociación entre la hepatitis grave, a veces mortal, y los regímenes antituberculosos que contienen isoniazida, pirazinamida y rifampicina está bien documentada. Recientemente se han descrito dos casos de insuficiencia hepática en pacientes con tuberculosis latente que estaban recibiendo tratamiento combinado con rifampicina y pirazinamida; uno de ellos falleció. El Programa Canadiense de Vigilancia de Reacciones Adversas a Fármacos $(\mathrm{Ca}$ nadian Adverse Drug Reaction Monitoring Program: CADRMP) examinó 420 notificaciones nacionales de sospecha de reacciones hepatobiliares adversas asociadas a diferentes combinaciones de isoniazida, rifampicina y pirazinamida, recibidas desde la fecha de su introducción en Canadá hasta el 18 de mayo de 2001. Estos informes indican que la hepatotoxicidad puede ocurrir en pacientes que reciban cualquiera de los fármacos por separado, o en dife- rentes combinaciones. Las normas canadienses sobre la tuberculosis recomiendan realizar pruebas funcionales hepáticas al iniciar el tratamiento con isoniazida, pero solo recomiendan la vigilancia regular en pacientes con hepatopatías preexistentes, antecedentes de abuso de alcohol o edad igual o superior a 35 años. La utilidad de la vigilancia de la función hepática en la detección de la insuficiencia hepática fulminante es controvertida y necesita ser mejor investigada. No obstante, se recuerda la importancia de la vigilancia de la hepatotoxicidad, mediante la clínica o la determinación de las enzimas hepáticas, durante el tratamiento con cualquier régimen antituberculoso. Es fundamental enseñar a los pacientes a detectar las manifestaciones sospechosas de hepatitis (náuseas, vómitos, gastralgia, falta de apetito, fatiga, coluria e ictericia) y recomendarles que dejen de tomar la medicación y consulten inmediatamente a su médico ante la aparición de estas manifestaciones.

\section{Efectos adversos cardiovasculares del rofecoxib (Canadá)}

Las autoridades sanitarias canadienses (Health Canada/Santé Canadá: HC/ SC) han informado a la población de la seguridad del rofecoxib (Vioxx $\left.{ }^{\circledR}\right)$, un antiinflamatorio no esteroideo (AINE) que inhibe selectivamente la ciclooxigenasa 2. En Canadá el rofecoxib necesita prescripción médica y está aprobado para el tratamiento de adultos con artrosis, dolor menstrual o dolor agudo. Los resultados de un ensayo clínico a gran escala tras la comercialización del fármaco han revelado que el riesgo de toxicidad gastrointestinal del rofecoxib es menos de la mitad que con el naproxeno, otro AINE. No obstante, se advierte a los pacientes de que en casos raros, especialmente en ancianos, se pueden producir problemas gástricos graves, tales como hemorragias. Los pacientes que presenten síntomas de toxicidad gastrointestinal, tales como gastralgia o sangre en heces, durante el tratamiento con rofecoxib deben consultar inmediatamente a su médico. Los resultados del mismo estudio han revelado también que la tasa de acontecimientos adversos cardiovasculares fue mayor en el grupo tratado con rofecoxib. Aunque todavía no se ha establecido una relación entre estos acontecimientos y el uso del fármaco, los pacientes con antecedentes de hipertensión, cardiopatía isquémica, retención de líquidos o insuficiencia cardíaca deben comentar estos hechos con su médico antes de empezar a tomar rofecoxib. Los pacientes que presenten retención de líquidos, disnea, debilidad, fatiga, gran aumento de peso o dolor torácico durante el tratamiento con rofecoxib deben informar inmediatamente a su médico.

\section{Trastornos hidroelectrolíticos con las soluciones orales de fosfato sódico (Canadá)}

Las soluciones orales de fosfato sódico se utilizan como laxantes y en el lavado intestinal previo a los exámenes radiográficos, a la colonoscopia y a las intervenciones quirúrgicas. Cuando no se utilizan apropiadamente, estas soluciones pueden causar trastornos hidroelectrolíticos (deshidratación y alteraciones de las concentraciones de calcio, fósforo, potasio y sodio), que a su vez pueden alterar la función cardíaca, muscular o renal. La dosis máxima recomendada en adultos es de $45 \mathrm{~mL}$ en 24 horas. Los pacientes con insuficiencia renal, cardiopatías, enteropatías, diarrea o vómitos, los que estén tomando otros fármacos que alteran las concentraciones de electrolitos, como los diuréticos, y los ancianos corren mayor riesgo de sufrir trastornos hidroelectrolíticos tras la toma de estas soluciones. Tanto en presencia como en ausencia de estos factores, el riesgo también aumenta en quienes tomen más de $45 \mathrm{~mL}$ en 24 horas. A los pacientes se les aconseja comentar sus antecedentes con el médico antes de tomar estos medicamentos y consultar inmediatamente en caso de que sufran síntomas de alteraciones hidroelectrolíticas, tales como hormigueo, entumecimiento, espasmos, debilidad muscular, temblores o 
palpitaciones. HC/SC seguirá examinando el perfil de seguridad de estas soluciones.

\section{Epoetina $\alpha \mathrm{y}$ aplasia pura de la serie roja (Canadá, Reino Unido)}

La epoetina $\alpha$ se usa en el tratamiento de la anemia asociada a la insuficiencia renal crónica, a la quimioterapia del cáncer, a la autodonación de sangre y a la cirugía ortopédica mayor electiva. En Canadá y en el Reino Unido se han descrito casos de anemia pura de la serie roja en pacientes con insuficiencia renal crónica tratados con epoetina $\alpha$. Generalmente, después de meses o años de tratamiento, los pacientes presentan un súbito empeoramiento de la anemia, que no responde al aumento de las dosis de epoetina $\alpha$ ni a ningún otro tipo de eritropoyetina, y se vuelven dependientes de las transfusiones de sangre. Por lo tanto, se advierte a los médicos de que deben vigilar la respuesta clínica a la epoetina $\alpha$. En pacientes que presenten una pérdida súbita de la eficacia del tratamiento o un empeoramiento de la anemia se deben investigar las causas típicas de ausencia de respuesta, como los déficit de hierro, folato o vitamina $B_{12}$, la intoxicación por aluminio, las infecciones y la inflamación, las pérdidas de sangre y la hemólisis. Ante la sospecha de anemia pura de la serie roja y en ausencia de otras causas, se deben realizar pruebas de detección de anticuerpos frente a la eritropoyetina y suspender inmediatamente la administración de epoetina $\alpha$. No se debe cambiar al tratamiento con ningún otro tipo de eritropoyetina, se excluirán otras causas de anemia pura de la serie roja y se instaurará un tratamiento apropiado.

\section{Trastornos hematológicos con la linezolida (Reino Unido)}

Desde su lanzamiento en el Reino Unido en enero del año 2001, la Agencia de Control de Medicamentos ( $\mathrm{Me}$ dicines Control Agency: MCA) de este país ha recibido 12 informes de tras- tornos hematológicos (trombocitopenia, anemia, leucocitopenia y pancitopenia) asociados a la linezolida (Zyvox ${ }^{\circledR}$ ). La mielosupresión es un efecto adverso reconocido de la linezolida y se recomienda una estrecha vigilancia en los siguientes pacientes:

- Los tratados durante más de 10 a 14 días.

- Los que ya tengan mielosupresión antes del inicio del tratamiento.

- Los que estén recibiendo otros tratamientos que puedan alterar la concentración de hemoglobina, la función plaquetaria o el hemograma.

- Los que padezcan insuficiencia renal.

La MCA advierte de que el tratamiento con linezolida se debe interrumpir en todo paciente que presente mielosupresión importante, a no ser que se considere esencial su continuación; en tal caso se debe iniciar una vigilancia intensiva y poner en práctica medidas clínicas apropiadas.

\section{Acidosis láctica mortal en pacientes con sida tratados con antirretrovíricos (Chile, Estados Unidos)}

Los inhibidores nucleosídicos de la retrotranscriptasa del virus de la inmunodeficiencia humana (VIH), y en particular la didanosina y la estavudina, se han asociado a casos graves de acidosis láctica e insuficiencia hepática. El mecanismo que explicaría esta reacción sería una acción directa sobre la ADN-polimerasa mitocondrial que provocaría la formación de ADN mitocondrial (ADNm) defectuoso, impidiendo así la formación de ATP mediante la fosforilación oxidativa y obligando a la célula a recurrir a la glucólisis anaerobia, que favorece la acumulación de lactato. La alteración del metabolismo mitocondrial llevaría también a la acumulación de triglicéridos en el hígado. En Chile y Estados Unidos (EE. UU.), las agencias reguladoras respectivas -el Instituto de Salud Pública y la Administración de Alimentos y Medicamientos (Food and Drug Administration: FDA) - han noti- ficado a los profesionales sanitarios del riesgo de acidosis láctica como complicación del tratamiento con estavudina o didanosina. En pacientes tratados con combinaciones de estavudina y otros antirretrovíricos se han descrito casos de debilidad neuromuscular ascendente de progresión rápida, con una presentación clínica similar a la del síndrome de Guillain-Barré, incluida la insuficiencia respiratoria. Algunos de estos casos fueron mortales y la mayoría de ellos se observaron en el contexto de acidosis láctica o hiperlactacidemia sintomática. Los signos y síntomas iniciales de hiperlactacidemia deberían recibir gran atención, puesto que su manifestación más extrema, el síndrome de acidosis láctica, es potencialmente mortal. En caso de que aparezca debilidad muscular en un paciente tratado con estavudina se debe suspender la administración del fármaco.

\section{Complicaciones tromboembólicas con el raloxifeno (Australia)}

El raloxifeno está aprobado en Australia, desde 1999, para el tratamiento de la osteoporosis posmenopáusica. Hasta la fecha, el Comité Asesor de Reacciones Adversas a Fármacos ( $A d-$ verse Drug Reactions Advisory Committee: ADRAC) ha sido notificado de 185 casos de sospecha de reacción adversa al raloxifeno. Muchas fueron leves (náuseas, sofocos, erupciones cutáneas, cefaleas, etc.) y están descritas en la información sobre el producto. Las más graves han consistido en trombosis venosa profunda (22 casos), embolia pulmonar (7 casos) y enfermedad cerebrovascular (10 casos). Se trataba de mujeres de 55 a 89 años (mediana de 71) con osteoporosis. Las reacciones ocurrieron desde algunos días hasta 8 meses después del inicio del tratamiento, pero generalmente se produjeron varios meses después. Una paciente falleció. Como el raloxifeno se asocia a un aumento del riesgo de tromboembolias venosas (TEV) comparable al del tratamiento de reposición hormonal, en pacientes con factores de riesgo de sufrir estos acon- 
tecimientos se debe evaluar cuidadosamente la relación entre los riesgos y los beneficios antes de iniciar el tratamiento. Se recomienda que las pacientes sean advertidas específicamente del aumento del riesgo de TEV que existe con todos los compuestos estrogénicos, incluido el raloxifeno. Además de TEV también se registraron siete casos de accidente cerebrovascular y tres de ataques isquémicos transitorios.

\section{Tramadol y síndrome serotonínico (Australia)}

El tramadol es un analgésico de acción central que se une a los receptores opioidérgicos, aunque no esté relacionado estructuralmente con los opioides. Además inhibe la recaptación de noradrenalina y serotonina. Desde su comercialización en Australia a finales de 1998, el ADRAC ha recibido 171 informes de sospecha de reacciones adversas, entre ellas 6 casos de síndrome serotoninérgico. En cuatro de estos casos los pacientes también estaban tomando antidepresivos que aumentan la concentración cerebral de serotonina: sertralina, citalopram, moclobemida y la combinación de amitriptilina y clomipramina. De los otros dos, uno estaba tomando hierba de San Juan, que también parece aumentar la concentración de serotonina, y el último correspondió a un anciano que estaba tomando una dosis de tramadol relativamente alta (400 mg/día). Se recomienda cautela al usar altas dosis de tramadol y al combinarlo con medicaciones que aumentan la concentración cerebral de serotonina.

\section{Toxicidad pulmonar de la amiodarona (Australia)}

El ADRAC fue notificado de 61 casos sospechosos de reacciones adversas a la amiodarona en el año 2000 y de otros 74, 5 de ellos mortales, en el 2001. Desde 1981 se han registrado en Australia 31 muertes asociadas al uso de este antiarrítmico, 17 de ellas relacionadas con causas pulmonares: fi- brosis pulmonar en 8 , infiltración pulmonar en 5 , neumonitis en 2 , derrame pleural en 1 e insuficiencia respiratoria en 1. Aunque generalmente es insidiosa, la toxicidad pulmonar causada por la amiodarona puede desarrollarse rápidamente. Se debe administrar la mínima dosis eficaz y los pacientes deben ser advertidos de la necesidad de consultar al médico ante la aparición de disnea o tos no productiva. Además, la amiodarona también puede producir hepatotoxicidad (cirrosis e insuficiencia hepática), efectos cardiovasculares (bradicardia y taquicardia), reacciones cutáneas (fotosensibilidad y coloración de la piel), neurotoxicidad (ataxia y neuropatía periférica), depósitos corneales e hipertiroidismo e hipotiroidismo.

\section{Reacciones adversas neuropsiquiátricas al zolpidem (Australia)}

El zolpidem se comercializó en Australia a finales del año 2000 para el tratamiento a corto plazo del insomnio. Aunque no está relacionado con las benzodiazepinas desde el punto de vista estructural, tiene una acción farmacológica similar. A lo largo del año 2001, el ADRAC recibió 72 informes en los que se describían 170 reacciones asociadas al zolpidem. De estos 72 informes, 56 describían una o más reacciones neurológicas o psiquiátricas, en especial alucinaciones visuales, confusión, depresión y amnesia. En su mayoría, estas reacciones ocurrieron con una dosis de $10 \mathrm{mg} /$ día, y el 70\% tras la primera toma. La mayoría de los 15 casos de alucinaciones ocurrieron a las pocas horas, a menudo poco después de la toma del medicamento. En la mayoría de los casos de amnesia hubo una pérdida total de la memoria de los acontecimientos inmediatamente posteriores a la toma del medicamento, aunque en dos hubo un deterioro de la memoria en los días siguientes. La confusión y la depresión aparecieron en algunos casos a las pocas horas de tomar el medicamento, pero en la mayoría ocurrieron al día siguiente. Los médicos deben saber que el zolpidem se puede asociar a reacciones neurológicas o psiquiátricas molestas.

\section{Cistitis con el ácido tiaprofénico (Nueva Zelandia)}

El Centro Neozelandés de Vigilancia de Reacciones Adversas (New Zealand Center for Adverse Reactions Monitoring) ha sido notificado en el año 2000 de dos casos de cistitis asociados al ácido tiaprofénico $\left(\right.$ Surgam $\left.^{\circledR}\right)$, 1o cual eleva el número total de casos a 17 y ha llevado a las autoridades a recordarles a los médicos del país la posibilidad de que se produzca esta complicación. En el Reino Unido, la tasa de notificación de casos de cistitis con este fármaco es de 18 por millón de prescripciones, frente a 0,05 a 0,2 por millón con otros antiinflamatorios no esteroideos. De cualquier forma, la cistitis es rara y puede ocurrir meses o años después del inicio del tratamiento; si no se identifica y no se suspende la medicación, puede llevar a intervenciones quirúrgicas innecesarias, a la producción de alteraciones permanentes de las vías urinarias y al deterioro de la función renal. Los ancianos en tratamiento prolongado con ácido tiaprofénico son especialmente vulnerables a la cistitis. Se recomienda usar el fármaco con cautela en pacientes con infecciones urinarias recurrentes, antecedentes de cistitis $\mathrm{u}$ otros síntomas urinarios, dado que pueden enmascarar los síntomas de la cistitis causada por el fármaco. Los pacientes en tratamiento con ácido tiaprofénico deben ser advertidos de la necesidad de buscar atención médica en caso de que presenten síntomas urinarios.

\section{Reacciones adversas al levofloxacino (Bélgica)}

El levofloxacino (Tavanic ${ }^{\circledR}$ ) se ha asociado a diferentes reacciones adversas, entre ellas 12 casos de tendinitis, notificadas al centro de farmacovigilancia de Bélgica desde el lanzamiento del fármaco en agosto del año 2000. De los 12 casos de tendinitis, en 6 hubo ro- 
tura de tendones; en 5 pudo haber contribuido el tratamiento simultáneo con corticoesteroides. Los pacientes tenían un promedio de 74 años y parece que el riesgo de tendinitis durante el tratamiento con levofloxacino aumenta con la edad. El centro también ha sido notificado de 8 casos de reacciones alérgicas (7 de edema angioneurótico y 1 de anafilaxia).

\section{USO RACIONAL}

\section{Nuevos consejos para la prescripción de propionato de fluticasona (Reino Unido)}

Se han actualizado los consejos para la prescripción del propionato de fluticasona inhalado, con el fin de minimizar el riesgo de efectos adversos sistémicos que se producen con las dosis elevadas. La información actualizada incluye las nuevas directrices siguientes, que aparecerán en la información sobre el producto de todos los preparados de propionato de fluticasona para inhalación.

- La dosis inicial debe ser proporcional a la gravedad de la enfermedad.

- La dosis debe reducirse gradualmente hasta alcanzar la mínima dosis que consiga controlar eficazmente el asma.

- Los pacientes con asma leve deben comenzar el tratamiento con una dosis de $100 \mu \mathrm{g}$ dos veces al día, mientras que aquellos con asma moderada a grave deben recibir una dosis inicial de 250 a $500 \mu \mathrm{g}$ dos veces al día.

- Solo se deben prescribir dosis $>500$ $\mu \mathrm{g}$ dos veces al día a pacientes con asma grave en los que se espere un beneficio clínico adicional y este quede demostrado por una mejoría de la función pulmonar o del control de los síntomas, o por una reducción de las dosis de corticoesteroides orales necesarias. Además, estas dosis solo deberían ser prescritas por médicos con suficiente experiencia en el tratamiento del asma.

\section{Seguridad gastrointestinal de los antiinflamatorios no esteroideos}

Un estudio realizado en Inglaterra en 1994 acerca de la seguridad gastrointestinal (GI) relativa de siete AINE distintos de la aspirina permitió identificar tres grandes grupos desde este punto de vista: los de alto riesgo (azapropazona), los de riesgo intermedio (piroxicam, indometacina, ketoprofeno, diclofenaco y naproxeno) y los de bajo riesgo (ibuprofeno). Los del primer grupo solo deberían usarse como fármacos de segunda línea para el tratamiento de la artritis reumatoidea, la espondilitis anquilosante y la gota aguda. El riesgo de perforación/obstrucción, ulceración o hemorragia gastrointestinal (PUHGI) aumenta a más del doble cuando se combina la aspirina con un AINE distinto de la aspirina. Estas combinaciones solo se deben usar cuando sean absolutamente necesarias y los pacientes deben ser vigilados cuidadosamente. El riesgo de toxicidad GI aumenta a medida que lo hacen las dosis de aspirina, pero no hay pruebas definitivas de que el riesgo cambie con diferentes formulaciones. Según los resultados de los ensayos clínicos, los nuevos AINE que inhiben selectivamente la ciclooxigenasa 2 (COX-2) tienen menor riesgo de PUHGI que los AINE no selectivos, pero también se han recibido notificaciones de PUHGI con el uso de estos fármacos. También es importante notar que el hecho de que se notifique una sospecha de reacción adversa no significa necesariamente que sea causada por el fármaco. Para un uso seguro de los AINE, se aconseja:

- Usar preferentemente fármacos con bajo riesgo de PUH GI, como el ibuprofeno.

- Iniciar el tratamiento con la menor dosis recomendada.

- No administrar simultáneamente más de un AINE.

- No usar AINE no selectivos en pacientes con antecedentes de úlcera péptica o úlcera péptica activa.

- No usar inhibidores de la COX-2 en pacientes con úlcera péptica activa.
- Solo usar combinaciones de aspirina y AINE distintos de la aspirina cuando sean absolutamente necesarias, puesto que parecen asociarse a un aumento del riesgo de toxicidad GI.

\section{ENMIENDAS A LA ROTULACIÓN/CAMBIOS DE FORMULACIÓN}

\section{Advertencias de uso en los prospectos del etanercept (Chile)}

En su resolución No. 5246 de 3 de julio de 2001, la Dirección del Instituto de Salud Pública de Chile ha determinado que los prospectos de información al profesional sobre los productos que contienen el principio activo etanercept deberán incluir advertencias sobre la notificación de casos de alteraciones desmielinizantes del sistema nervioso central y pancitopenia, entre ellos algunos de anemia aplásica.

\section{Advertencia a los profesionales del uso de oprelvekina en pacientes pediátricos (EE. UU.)}

Wyeth-Ayerst ha enviado a los profesionales sanitarios una carta en la que les informa de importantes cambios en la información sobre la seguridad de la oprelvekina (Neumega ${ }^{\circledR}$ ) en pacientes pediátricos. Se señala que no hay estudios controlados que hayan determinado la dosis de oprelvekina eficaz y segura en niños. Por lo tanto, se recomienda que el fármaco solo se administre a estos pacientes, y en especial a los menores de 12 años, en ensayos clínicos controlados y bajo una estrecha vigilancia. La carta también manifiesta que en un estudio farmacocinético se ha identificado el papiledema como una reacción adversa limitante de la dosis de oprelvekina. En este estudio de 47 pacientes pediátricos, 4 de los 16 que recibieron 100 $\mu \mathrm{g} / \mathrm{kg} /$ día de oprelvekina sufrieron papiledema, complicación que no se observó en ninguno de los 9 tratados con $75 \mu \mathrm{g} / \mathrm{kg} /$ día; sin embargo, de- 
bido al pequeño número de casos tratados con esta dosis, la verdadera incidencia podría llegar al $33 \%$. Por otra parte, los menores de 12 años tratados con $50 \mu \mathrm{g} / \mathrm{kg} /$ día no alcanzaron una concentración sérica del fármaco que se pudiera considerar eficaz. Aunque estos datos son preliminares, la carta de Wyeth-Ayerst menciona que, una vez que se realicen estudios completos, se modificará el prospecto para incluir toda la información relacionada con la administración de la oprelvekina a pacientes pediátricos.

\section{Cambios en la información sobre la seguridad de Intron ${ }^{\circledR}$ A interferón $\left(\alpha_{2 b}\right)$ (EE. UU.)}

Recientemente se han producido cambios en la información sobre la seguridad incluida en los prospectos de los medicamentos Intron ${ }^{\circledR}$ A (interferón $\alpha_{2 b}$ recombinante) para inyección y Rebetron ${ }^{\circledR}$, consistente en la combinación de Rebetol ${ }^{\circledR}$ (ribavirina) cápsulas e Intron ${ }^{\circledR}$ A. Los cambios incluyen la adición de una advertencia enmarcada sobre la aparición de trastornos neuropsiquiátricos, autoinmunitarios, isquémicos e infecciosos en pacientes tratados con Intron ${ }^{\circledR}$ A. La versión revisada de la advertencia también menciona los requisitos específicos de vigilancia de los pacientes para detectar estos acontecimientos potencialmente mortales. Esta advertencia ha sido añadida a la información de todos los productos que contienen interferones $\alpha$.

\section{Cambios en los prospectos de la pioglitazona y la rosiglitazona (EE. UU.)}

La FDA ha aprobado cambios en las secciones de "Advertencias", "Precauciones" y "Reacciones adversas" de los prospectos de la pioglitazona y la rosiglitazona, con el fin de dejar más claros los riesgos cardiovasculares de estas tiazolidinedionas, tanto en monoterapia como en combinación con otros antidiabéticos, y en particular con la insulina.

\section{Solicitud de revisión del prospecto de Exelon ${ }^{\circledR}$ (rivastigmina) (Brasil)}

El tartarato de rivastigmina (Exe$\operatorname{lon}^{\circledR}$ ), aprobado por la FDA en abril del año 2000, es un inhibidor de la acetilcolinesterasa que, como tal, actúa retrasando la degradación de la acetilcolina liberada por las neuronas colinérgicas funcionalmente intactas, con lo cual aumenta la neurotransmisión colinérgica. En la enfermedad de Alzheimer, los inhibidores de la colinesterasa tienen una acción esencialmente sintomática y no hay pruebas de que retrasen la progresión de la enfermedad. En cerca del 10\% de los pacientes producen una importante mejoría clínica del funcionamiento cognitivo, pero en aproximadamente un $20 \%$ de estos pacientes producen efectos adversos colinérgicos previsibles, generalmente al principio del tratamiento, que llevan a la interrupción del mismo. Se aconseja que el tratamiento solo sea iniciado, evaluado y continuado por neurólogos, geriatras o psiquiatras. El tratamiento se debe interrumpir ante la aparición de acontecimientos adversos importantes, mal cumplimiento del mismo o deterioro significativo del estado clínico del paciente. Todo paciente tratado con rivastigmina debe ser examinado semanalmente durante las primeras 6 semanas de tratamiento o cada vez que se modifique la dosis. Posteriormente, debe ser examinado al menos una vez cada 6 meses. Aunque el prospecto del medicamento afirma que en los ensayos clínicos realizados antes de su comercialización no hubo interacciones farmacocinéticas ni aumento de las reacciones adversas clínicamente relevantes al asociar la rivastigmina con digoxina, warfarina, fluoxetina, antiácidos, antieméticos, hipoglucemiantes, antihipertensores de acción central, betabloqueantes, bloqueantes de los canales del calcio, inotrópicos, antianginosos, antiinflamatorios no esteroideos, estrógenos, analgésicos, benzodiazepinas $\mathrm{o}$ antihistamínicos, de las 23 notificaciones de sospecha de reacciones adversas a la rivastigmina recibidas por el Sector de Farmacovigilancia del Centro de
Vigilancia Sanitaria del Estado de São Paulo, solo en 5 no se mencionaba su asociación a otros medicamentos. Por lo tanto, el mencionado organismo recomienda extremar la cautela al indicar la rivastigmina en pacientes que estén tomando cualquiera de esos fármacos, independientemente de lo que diga el prospecto. Además, le ha solicitado al fabricante (Novartis Biociências, S.A.) que revise el prospecto de Exelon ${ }^{\circledR}$ vigente en Brasil y concluye que son necesarios nuevos estudios para excluir realmente la existencia de interacciones medicamentosas.

\section{EVALUACIÓN DE NUEVOS PRODUCTOS}

\section{Usos y efectos indeseables de los nuevos hipoglucemiantes orales (España)}

En los últimos años han aparecido nuevos fármacos para el tratamiento de la diabetes, como la acarbosa, el miglitol, la pioglitazona y la rosiglitazona. Por otra parte, los resultados de estudios clínicos recientes han originado modificaciones del tratamiento, entre ellas la prescripción de metformina a muchos pacientes que no la recibían antes.

Las tiazolidindionas son un nuevo grupo de hipoglucemiantes orales con un mecanismo de acción parecido al de las biguanidas. Disminuyen la neoglucogénesis hepática y aumentan la sensibilidad del músculo y del tejido adiposo a la insulina, pero, al contrario de las sulfonilureas, no aumentan la secreción de insulina. La primera tiazolidindiona, la troglitazona, fue retirada del mercado poco después de su comercialización debido a casos graves de hepatotoxicidad. Recientemente, la EMEA ha autorizado otras dos tiazolidindionas, la pioglitazona y la rosiglitazona, para el tratamiento de pacientes que no se normalicen con monoterapia. Está aprobado su uso combinado con metformina en pacientes obesos, o con una sulfonilurea en casos de intolerancia o contraindicación de la metformina. 
El efecto indeseado más frecuente de las tiazolidindionas es la disminución de la hemoglobinemia y del hematocrito, que parece deberse al aumento del volumen plasmático y no suele producir síntomas de anemia. La notificación de varios casos de descompensación de la insuficiencia cardíaca con la troglitazona, la rosiglitazona y la pioglitazona es uno de los motivos de que las tiazolidindionas estén contraindicadas en pacientes con insuficiencia cardíaca y de que se recomiende una rigurosa vigilancia de los pacientes con reservas cardíacas disminuidas.

Por otro lado, las tiazolidindionas alteran el metabolismo lipídico porque aumentan la degradación de los triglicéridos. La rosiglitazona aumenta las concentraciones de colesterol total y de las lipoproteínas de baja y alta densidad (LDL y HDL) en un 10 a $20 \%$, pero el cociente entre el colesterol total y el colesterol de las HDL no se modifica o solo disminuye ligeramente; los cambios de los triglicéridos plasmáticos son muy variables. Con la pioglitazona se han observado menores aumentos del colesterol de las LDL y disminuciones de los triglicéridos. Son necesarios nuevos estudios a largo plazo para determinar si estos cambios del perfil lipídico tienen repercusiones sobre la morbimortalidad cardiovascular.

Aunque inicialmente se consideró que la hepatotoxicidad no era un efecto de clase, la pioglitazona y la rosiglitazona tienen una estructura muy similar a la troglitazona y, de hecho, ya se han descrito casos de aumento moderado de las transaminasas y algún caso de insuficiencia hepática con la rosiglitazona. Por lo tanto, se recomienda determinar la alanina-aminotransferasa (ALT) antes de iniciar el tratamiento con estos fármacos y vigilar al paciente cada 2 meses durante el primer año de tratamiento. En caso de ictericia o aumento de la ALT a más de tres veces su valor normal se debe retirar el fármaco.

Los resultados de un estudio clínico (United Kingdom Prospective Diabetes Study) revelaron que el tratamiento con metformina proporciona una re- ducción significativa de las complicaciones microvasculares y de la mortalidad global en pacientes obesos. Esto ha producido un aumento del número de pacientes tratados con este fármaco $\mathrm{y}$, por consiguiente, de la posibilidad de observar efectos adversos. Estos son potencialmente graves, pero prevenibles, siempre que se tomen ciertas precauciones.

La metformina, como las demás biguanidas, disminuye la neoglucogénesis hepática y aumenta la sensibilidad del tejido muscular a la insulina. Las reacciones adversas más frecuentes son de carácter digestivo (dispepsia y diarrea). Además puede interferir con la absorción de la vitamina $B_{12}$, pero este efecto raramente tiene repercusiones clínicas. El efecto más grave, que ha limitado su uso, es la acidosis metabólica, cuya letalidad puede llegar al $50 \%$. Afortunadamente se trata de un efecto raro (5 a 9 casos / 100000 pacientes-año), sobre todo en ausencia de factores desencadenantes o contribuyentes. La insuficiencia renal, las situaciones agudas de hipoperfusión, la hipoxemia y las alteraciones graves de la función hepática favorecen la aparición de acidosis láctica por biguanidas y contraindican formalmente su empleo. Con respecto a la insuficiencia renal, que tiene una elevada prevalencia en pacientes diabéticos, la metformina está contraindicada cuando el aclaramiento de creatinina es $<70,2$ $\mathrm{mL} / \mathrm{min}$. En pacientes con masa muscular reducida, como los ancianos, la concentración plasmática de creatinina puede subestimar la filtración glomerular, por lo que es necesario determinar el aclaramiento de creatinina. La metformina también está contraindicada en pacientes con insuficiencia cardíaca, en los que es de suponer que hay una disminución de la perfusión renal.

\section{La FDA aprueba el primer anticonceptivo hormonal en parches cutáneos (EE. UU.)}

La FDA ha aprobado el primer parche transdérmico (Ortho Evra ${ }^{\circledR}$ ) para ser usado como anticonceptivo. El par- che consta de tres capas, una de ellas adhesiva, que es la que libera lentamente las dos hormonas (norelgestromin y etinilestradiol) a través de la piel hacia la circulación. El parche puede aplicarse en la parte baja del abdomen, las nalgas o el tórax, pero no en las mamas. El tratamiento consiste en la aplicación de un parche durante cada una de las tres primeras semanas del ciclo y los cambios deben efectuarse siempre el mismo día de la semana. En la cuarta semana del ciclo no se aplica ningún parche, lo cual permite que la mujer tenga la menstruación, como si estuviera tomando anticonceptivos orales (AO). Al igual que los $\mathrm{AO}$, estos parches son eficaces para evitar el embarazo, siempre que se usen como se indica. Los riesgos son similares a los de los $\mathrm{AO}$, que son, entre otros, un aumento del riesgo de fenómenos trombóticos, ataques cardíacos y accidentes cerebrovasculares. El prospecto también señala que el consumo de tabaco aumenta el riesgo de efectos colaterales cardiovasculares graves. Se han realizado tres ensayos clínicos de alcance mundial con un total de 4578 participantes, de las cuales 3319 usaron Ortho Evra ${ }^{\circledR}$. Las demás usaron $\mathrm{AO}$ (grupo testigo). Estos ensayos clínicos demostraron una buena adherencia al tratamiento e indicaron que los parches pueden ser menos eficaces en mujeres con más de $90 \mathrm{~kg}$ de peso. En cerca del $5 \%$ de las mujeres hubo al menos un parche que se desprendió y cerca del $2 \%$ de las participantes abandonaron el estudio debido a que los parches les produjeron irritación cutánea.

\section{POLÍTICAS DE MEDICAMENTOS}

\section{Conferencia Panamericana sobre Armonización de la Reglamentación Farmacéutica}

En abril de 2002 se celebró en Washington, D.C., la III Conferencia Panamericana sobre Armonización de la Reglamentación Farmacéutica, en la que participaron las autoridades reguladoras de medicamentos de la mayoría de los países del continente americano, representantes de asociaciones 
de consumidores, de la industria farmacéutica y de instituciones educativas, entre otros. Son objetivos de esta serie de conferencias periódicas:

- Promover y mantener un diálogo constructivo entre las agencias reguladoras, la industria farmacéutica y otros sectores.

- Promover la convergencia de los sistemas de regulación de medicamentos en la Región.

- Adoptar recomendaciones para su implementación en el ámbito nacional y regional.

- Promover la armonización de los requisitos y regulaciones farmacéuticas en aspectos específicos.

Para desarrollar estos objetivos se les dio seguimiento a los Grupos Técnicos en áreas prioritarias de los procesos de armonización: buenas prácticas de fabricación y buenas prácticas clínicas, combate de la falsificación de medicamentos, bioequivalencia y farmacopeas.

Entre las recomendaciones generales formuladas por la III Conferencia se encuentran:

- Armonizar los Códigos de Ética y del Consentimiento Informado para las investigaciones clínicas en la Región.

- Priorizar medicamentos para la realización de ensayos de bioequivalencia y definición de sus productos de referencia.

- Reforzar una vez más la implementación de las buenas prácticas de fabricación, dando seguimiento a las acciones ya iniciadas.

\section{La OMS lanza la primera estrategia mundial sobre medicina tradicional y alternativa}

En los países en desarrollo, la medicina tradicional es usada como parte de la atención primaria por más del $80 \%$ de la población, y en los países industrializados cada vez son más numerosos los pacientes que recurren a la medicina alternativa con fines preventivos o paliativos. Así, por ejemplo, en Francia el $75 \%$ de la población ha usado la medicina complementaria al menos una vez; en Alemania, el 77\% de las clínicas del dolor proporcionan servicios de acupuntura, y en el Reino Unido el gasto anual en medicina complementaria o alternativa asciende a 2300 millones de dólares estadounidenses. Pero el uso incorrecto de los tratamientos tradicionales puede causar problemas. Por ejemplo, la hierba Ma huang (Ephedra) se ha utilizado tradicionalmente en China en el tratamiento a corto plazo de la congestión respiratoria, pero en los EE. UU., donde se comercializó como suplemento dietético, su uso a largo plazo se ha asociado al menos a una docena de muertes, ataques cardíacos y accidentes cerebrovasculares. En Bélgica, al menos 70 personas han necesitado trasplantes renales o diálisis por fibrosis intersticial después de haber tomado, también como suplemento dietético, hierbas de la familia Aristolochiaceae.

"La medicina tradicional o complementaria es víctima tanto de entusiastas acríticos como de escépticos desinformados", explicó el Dr. Yasuhiro Suzuki, Director Ejecutivo del grupo orgánico de Tecnología de la Salud y Productos Farmacéuticos de la Organización Mundial de la Salud (OMS). En los países en desarrollo, donde más de un tercio de la población no tiene acceso a los medicamentos esenciales, la provisión de tratamientos eficaces y seguros de la medicina tradicional y de la medicina complementaria o alternativa (MT/MCA) podría convertirse en una herramienta crítica para aumentar el acceso a la atención sanitaria. Pero aunque la medicina tradicional se ha integrado plenamente en los sistemas de salud de China, Corea del Norte, Corea del Sur y Vietnam, todavía no ha ocurrido lo mismo en muchos otros países.

El mercado mundial de los tratamientos tradicionales representa una cifra de 60 mil millones de dólares estadounidenses y sigue creciendo. Además del problema de la seguridad de los pacientes y de la amenaza al conocimiento y a la biodiversidad, también hay que tener en cuenta el riesgo de que su comercialización no regulada haga que muchos de aquellos que dependen de estos tratamientos como principal fuente de atención sanitaria no puedan pagarlos. Es por ello que son necesarias políticas de protección de los conocimientos indígenas o tradicionales.

Esta situación ha suscitado cierta preocupación entre los profesionales y los consumidores, sobre todo con respecto a la seguridad de estas prácticas, pero también con respecto a cuestiones de políticas, regulaciones, pruebas, biodiversidad y conservación y protección del saber tradicional. La OMS ha lanzado una estrategia mundial para abordar estos problemas cuyo objetivo consiste en proporcionar un marco para la política de asistencia a los países que les ayude a:

- Desarrollar políticas nacionales de evaluación y regulación de las prácticas de la MT/MCA.

- Fortalecer la base de pruebas sobre la seguridad, eficacia y calidad de los productos y prácticas de la MT/ MCA.

- Garantizar la disponibilidad y la capacidad de comprar dichos productos y prácticas, entre ellos las hierbas medicinales esenciales.

- Promover el uso terapéutico fundamentado de la MT/MCA por parte tanto de los proveedores como de los consumidores.

\section{La anticoncepción de emergencia estará disponible sin necesidad de prescripción médica (Nueva Zelandia)}

La autoridad neozelandesa responsable de la seguridad de los medicamentos y aparatos médicos (Medsafe) ha comunicado que los anticonceptivos de emergencia a base de levonorgestrel podrá ser dispensada por enfermeras tituladas y farmacéuticos sin necesidad de prescripción médica. La anticoncepción de emergencia, conocida también como píldora del día siguiente, se usa para evitar el embarazo en las 72 horas después de una relación sexual sin protección con anticonceptivos. Esta decisión facilitará el acceso a la anticoncepción de emergencia, con el fin de reducir el número de embarazos no 
deseados y abortos. El Ministerio de Salud está trabajando con el Consejo de Enfermería y la Sociedad de Farmacéuticos para que el sistema que permita esta venta esté en funcionamiento a finales del año 2001 o a comienzos del 2002. Los anticonceptivos de emergencia ya se pueden vender sin necesidad de receta médica en otros países, como Francia, Noruega, el Reino Unido y algunas regiones de Canadá.

\section{REFERENCIAS}

TGA. Australian Adverse Drug Reactions Bulletin 2001;20(4):2-3.

TGA. Australian Adverse Drug Reactions Bulletin 2002;21(1):2-3.

CEMINEF/Instituto de Salud Pública de Chile. Boletín Informativo sobre Medicamentos 2001;18(2-3):16-18.

Committee on Safety of Medicines/MCA. Current Problems in Pharmacovigilance 2002;28 (April):2-5
FDA Talk Paper, November 2001.

Fundació Institut Català de Farmacologia. Butlletí Groc. 2001;14(2):5-7.

WHO Pharmaceuticals Newsletter 2002;(1):1-14.

WHO Drug Information 2001;15(3-4):154-155.

WHO Press Releases WHO/38. 16 de mayo de 2002. Disponible en: http://www.who.int/inf/ en/pr-2002-38. Acceso el 12 de junio de 2002.

ANVISA Alerta Ufarm/Anvisa No. 07, 20/12/01. Disponible en http://www.anvisa.gov.br/ farmacovigilancia/alerta / federal/2002/ federal_7_02.htm Acceso el 12 de junio de 2002.

ANVISA Alerta CVS-SP No. 04/02. Disponible en http://www.anvisa.gov.br/farmacovigilancia/ alerta/estadual/estadual_4.htm. Acceso el 12 de junio de 2002.

FDA/MEDWATCH, 2002. Safety Information Summaries. Disponible en http://www.fda. gov/medwatch/SAFETY/2002/safety02.htm \#drugs. Acceso el 12 de junio de 2002.

Health Canada Online, 2002. Warnings/Advisories. Disponible en http://www.hc-sc.gc.ca/ english/protection/warnings/2002.htm. Acceso el 12 de junio de 2002.

Ministerio della Salute. Comunicato No. 64 06/03/2002. Disponible en: http://www. sanita.it/sanita/farmaci/note_informative/ sibutramina/documenti/comunicatostampa1. pdf. Acceso el 12 de junio de 2002.
Información farmacológica da a conocer las decisiones oficiales sobre regulación de productos farmacéuticos adoptadas por organismos gubernamentales e internacionales en todo el mundo, los fundamentos científicos en que se sustentan tales decisiones y otros datos de interés relacionados con el tema. Como la mayor parte de la información proviene de fuentes de circulación relativamente limitada, su diseminación en esta forma permite hacerla llegar a un público más amplio. De esta manera se pretende contribuir a limitar el uso irracional de medicamentos y fomentar su uso racional en la Región de las Américas. La sección está a cargo del Programa de Medicamentos Esenciales y Tecnología para la Salud (HSE) de la OPS y se publica en la Revista Panamericana de Salud Públical Pan American Journal of Public Health con regularidad. Las separatas pueden solicitarse al programa mencionado, Organización Panamericana de la Salud, 525 Twenty-third Street, NW, Washington, DC 20037, EUA

\section{Pruebas básicas para medicamentos: sustancias farmacéuticas, plantas medicinales y formas farmacéuticas}

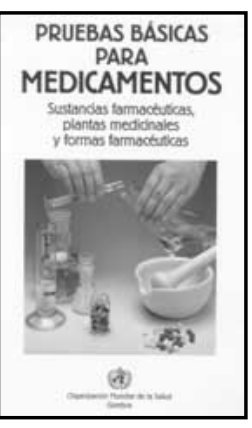

1999, $91 \mathrm{pp}$.

ISBN 9243545132 Código: WHO 20

Precio: US\$17.00/

US\$ 12.00 en América Latina y el Caribe
Este manual, que es complemento de Pruebas básicas para sustancias farmacéuticas y Pruebas básicas para formas farmacéuticas, presenta técnicas sencillas y fáciles de realizar para verificar la identidad de cierto número de sustancias y formas farmacéuticas, así como de plantas medicinales de uso común. Describe asimismo pruebas para detectar signos claros del deterioro de sustancias poco estables. Los métodos descritos se basan en el uso de un número limitado de reactivos y de instrumentos fáciles de encontrar, y no requieren un laboratorio plenamente equipado. Tampoco es necesario que los aplique un farmacéutico habilitado, pero sí una persona con algún conocimiento de química analítica.

Estas pruebas básicas no están en absoluto destinadas a sustituir las indicadas en las monografías farmacopeicas, que ofrecen una garantía de la calidad y no meramente una confirmación de la identidad. 